\title{
Forming of Ultrashort Electromagnetic Clusters by Two-Stream Superheterodyne Free Electron Lasers
}

\author{
V.V. Kulish ${ }^{a}$, A.V. Lysenko ${ }^{b, *}$, M.Yu. Rombovsky $^{b}$, V.V. Koval ${ }^{b}$ And I.I. VolK ${ }^{b}$ \\ ${ }^{a}$ National Aviation University, Department of Theoretical Physics, Kiev, 03058, Ukraine \\ ${ }^{b}$ Sumy State University, Department of General and Theoretical Physics, Sumy, 40007, Ukraine
}

(Received June 27, 2015; in final form July 15, 2016)

We construct a cubic nonlinear self-consistent theory of multiharmonic two-stream superheterodyne free electron lasers of a klystron type, intended to form powerful ultrashort clusters of an electromagnetic field. Plural three-wave parametric resonant interactions of wave harmonics have been taken into account. The two-stream superheterodyne free electron lasers mode in which the first harmonic of a space-charge wave is much smaller than the critical frequency of two-stream instability has been used for forming of the powerful ultrashort clusters of electromagnetic waves. We carry out amplitude, phase and spectral analyses of the processes occurring in such devices. We found out the conditions necessary for the forming of the ultrashort clusters of an electromagnetic field. We demonstrate the possibility of the ultrashort electromagnetic cluster formation in the multiharmonic two-stream superheterodyne free electron lasers-type systems.

DOI: 10.12693/APhysPolA.131.213

PACS/topics: 41.60.Cr

\section{Introduction}

Two-stream superheterodyne free electron lasers (TSFELs) are characterized by exceptionally high rates of the amplification of electromagnetic waves in the submillimeter-IR range [1-18]. Such high rates of the amplification are achieved through the use of the additional amplification mechanism of space-charge wave (SCW) due to the two-stream instability [19-21]. Therefore, at the same system parameters (currents, beam energies, amplitudes of a pump field, etc.) as in the conventional FEL, the identical amplification of the signal wave in TSFEL is achieved at significantly smaller length of the system. We should also note SCW that grow due to the two-stream instability are characterized by linear dispersion dependences [19-21]. Therefore the satisfaction of three-wave parametric resonance conditions becomes possible for the harmonics of such waves. The mentioned above feature of wave dispersion relations leads to simultaneous excitation of many harmonics. As a result, TSFEL operating modes, in which tens and more harmonics with comparable amplitudes are excited become possible [11,21-23]. Due to this property, there is a possibility of the generation of ultrashort intense clusters of an electromagnetic field on the basis of such multiharmonic TSFEL as a result of the superposition of many harmonics. Such ultrashort electromagnetic clusters, as well as ultrashort pulses can have wide practical application for a number of fundamental and applied researches in physics, chemistry, biology, medicine [24, 25].

This article focuses on the study of the processes occurring in the multiharmonic TSFEL-klystron, which are intended to form ultrashort clusters of an electromagnetic

\footnotetext{
*corresponding author; e-mail: lysenko_@ukr.net
}

field, and on clarification of conditions necessary for the creation of such clusters. Earlier multiharmonic properties of TSFEL were investigated in [11,21-23]. In paper [11] the method of forming of ultrashort electromagnetic field clusters as a result of multiharmonic signal superposition was proposed. The principal possibility of development of such systems on the basis of TSFEL was shown. In Refs. [22, 23] the analysis of plural three-wave interactions between harmonics of SCW in the development of two-stream instability was carried out. Such instability is used in the multiharmonic TSFEL. A detailed analysis of multiharmonic SCW energy transformation processes into the electromagnetic signal has not been made previously. In the presented study we carry out such analysis and find out the conditions necessary for short electromagnetic cluster formation in TSFEL. We demonstrate the possibility of creating of ultrashort electromagnetic clusters in systems such as multiharmonic TSFEL.

2. Model of multiharmonic two-stream superheterodyne FEL of a klystron type

A multiharmonic TSFEL-klystron scheme is presented in Fig. 1. Let us carry out the qualitative analysis of the basic principles of its operation.

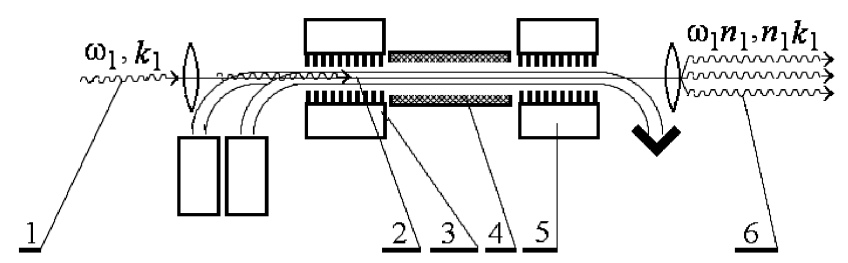

Fig. 1. Scheme of the multiharmonic TSFEL-klystron.

Monochromatic electromagnetic signal 1 with frequency $\omega_{1}$ and wave number $k_{1}$ is entering the working bulk of modulator section 3 . Two-stream electron beam 2 
is also fed here. The main purpose of modulator section 3 in TSFEL is excitement of two space-charge waves in the bulk of the two-stream beam. Modulator 3 can have several different designs. In this paper we consider the H-ubitron model of undulator. Since the monochromatic signal 1 is at the input of modulator 3 , we choose the modulator field monochromatic. The monochromatic SCW with frequency $\omega_{3,1}$ is excited in the two-stream electron beam as a result of the three-wave parametric resonance between monochromatic signal wave 1 and monochromatic modulator field 3. Next, modulated electron beam 2 enters in transit section 4 . Electromagnetic signal wave 1 at the input of this section is absorbed.

The main purpose of transit section 4 is amplification and intense excitement of SCW higher harmonics due to the two-stream instability. For this purpose we choose the frequency $\omega_{3,1}$ of the first SCW harmonic much less than the critical frequency of two-stream instability $\omega_{\mathrm{cr}}$. This ensures that all harmonics whose frequency is less than the critical frequency $\omega_{\text {cr }}$ will be amplified due to the two-stream instability. In this case the growth increment of SCW harmonics will increase with the harmonic number up to the optimal frequency $\omega_{\text {opt }}=\sqrt{3 / 8} \omega_{\mathrm{cr}} \approx 0.61 \omega_{\mathrm{cr}}$, which corresponds to the maximum growth increment. Another feature of SCWs growing due to two-stream instability is the fact that such waves are characterized by linear dispersion relations. Therefore, the set of three-wave parametric resonance interactions arise between the SCW harmonics. This leads to the intense excitation and amplification of SCW harmonics due to the three-wave parametric resonances. Thus, there is intensive generation and amplification of SCWs in the transit section due to the three-wave resonant interactions as well as the two-stream instability. As a result, SCW with a broad frequency spectrum is formed at the output of transit section 4 . Such spectrum contains an abnormal area where higher SCW harmonics have higher amplitudes.

After passing through transit section 4 the modulated beam is directed to the input of terminal section 5 . In this section there is a multiharmonic $\mathrm{H}$-ubitron field. The generation of the multiharmonic electromagnetic signal 5 and its further amplification occurs due to parametric resonances between SCW harmonics and harmonics of multiharmonic pumping field. In fact, the opposite conversion of multiharmonic SCW energy to multiharmonic signal 5 energy takes place. In addition, for each of the multiharmonic SCW harmonics the condition of parametric resonance with the corresponding harmonics of the pump field and the electromagnetic signal is satisfied. By adjusting the shape of the SCW spectrum and the parameters of multiharmonic pump field, we get the opportunity to control the multiharmonic spectrum of electromagnetic signal 6 at the output of TSFEL and we are able to form the ultrashort cluster of an electromagnetic field.

Comparing the electron beam modulation section and terminal section, we see that from a formal point of view, they can be considered similar. The main differences between them relate mainly to boundary conditions. In the modulation section amplitudes of all SCW harmonics are zero, and amplitude of at least one signal harmonic is non-zero. We have opposite situation in the terminal section. Namely, at the input the SCW harmonics are non-zero, when all signal harmonics are zero. The signal harmonics are generated in the process of wave interactions in the working bulk of the terminal section.

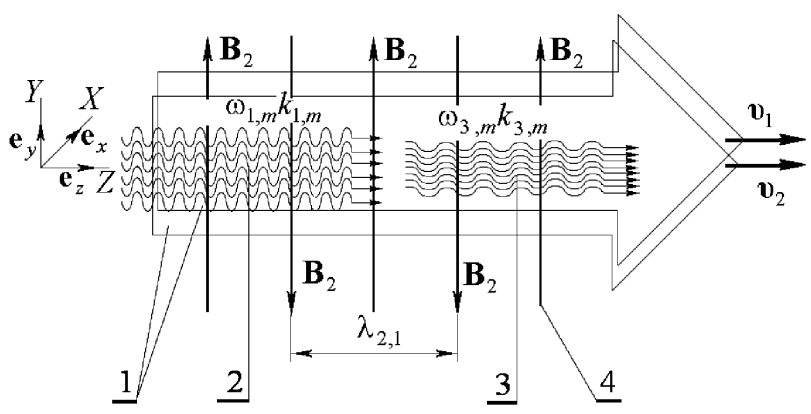

Fig. 2. Theoretical model of the terminal section and the modulator.

Therefore, for the modulation section as well as for the terminal section we use the theoretical model shown in Fig. 2. Here the two-stream electron beam 1 with partial velocities $\boldsymbol{v}_{1}, \boldsymbol{v}_{2}$ passes through the undulator, in which there is a multiharmonic pumping magnetic field 4 with induction $\boldsymbol{B}_{2}$ and undulation period $\lambda_{2,1}$. In the same region multiharmonic transverse electromagnetic signal 2 with electric field strength $\boldsymbol{E}_{1}$ and magnetic field induction $\boldsymbol{B}_{1}$ move along the axis $Z$. Multiharmonic SCW 3 with electric field strength $\boldsymbol{E}_{3}$ also moves along the axis $Z$.

Since all the waves are multiharmonic, strength $\boldsymbol{E}_{1}$ and induction $\boldsymbol{B}_{1}$ of the electromagnetic signal wave, induction $\boldsymbol{B}_{2}$ of the pumping field, and electric field strength $\boldsymbol{E}_{3}$ of the growing longitudinal SCW we represent in the form

$$
\begin{aligned}
& \boldsymbol{E}_{1}=E_{1} \boldsymbol{e}_{x}=\sum_{m=1}^{N}\left[E_{1, m} \exp \left(\mathrm{i} p_{1, m}\right)+\text { c.c. }\right] \boldsymbol{e}_{x}, \\
& \boldsymbol{B}_{1}=B_{1} \boldsymbol{e}_{y}=\sum_{m=1}^{N}\left[B_{1, m} \exp \left(\mathrm{i} p_{1, m}\right)+\text { c.c. }\right] \boldsymbol{e}_{y}, \\
& \boldsymbol{B}_{2}=B_{2} \boldsymbol{e}_{y}=\sum_{m=1}^{N}\left[B_{2, m} \exp \left(\text { i } p_{2, m}\right)+\text { c.c. }\right] \boldsymbol{e}_{y}, \\
& \boldsymbol{E}_{3}=E_{3} \boldsymbol{e}_{z}=\sum_{m=1}^{N}\left[E_{3, m} \exp \left(\text { i } p_{3, m}\right)+\text { c.c. }\right] \boldsymbol{e}_{z} .
\end{aligned}
$$

In relations (1) $E_{1, m}, E_{3, m}, B_{1, m}, B_{2, m}$ are the amplitudes of $m$-th harmonics of corresponding waves, $\boldsymbol{e}_{x}, \boldsymbol{e}_{y}, \boldsymbol{e}_{z}$ are the unit vectors of corresponding axis

$$
p_{\chi, m}=\omega_{\chi, m} t-k_{\chi, m} z=m \omega_{\chi, 1} t-k_{\chi, m} z
$$
are their phases ( $\chi$ index has values 1,2 , and 3 ), $\omega_{\chi, m}=$ 
$m \omega_{\chi, 1}$ are their frequencies $\left(\omega_{2,1}=0\right)$, and $k_{\chi, m}$ are their wave numbers. Thus, the electric and magnetic fields in the studied sections of TSFEL-klystron have the form

$$
\boldsymbol{E}=\boldsymbol{E}_{1}+\boldsymbol{E}_{3}, \quad \boldsymbol{B}=\boldsymbol{B}_{1}+\boldsymbol{B}_{2} .
$$

\section{Plural three-wave parametric resonances}

In the considered system there are three groups of plural three-wave parametric resonant interactions. Firstly, there are plural parametric resonances between SCW harmonics $[22,23]$. As known, growing SCW is characterized by linear dispersion relation [19-21]. It means that for any $m$-th harmonic of the wave the frequency $\omega_{3, m}$ and the wave number $k_{3, m}$ are related with the frequency $\omega_{3,1}$ and the wave number $k_{3,1}$ of the first harmonic as follows:

$$
\omega_{3, m}=m \omega_{3,1}, \quad k_{3, m}=m k_{3,1} .
$$

It leads to the fact that in order to $m_{1}$-th, $m_{2}$-th, and $m_{3}$ th SCW harmonics interacting with each other through a three-wave parametric resonance mechanism their numbers must be related by relation

$$
m_{1}=m_{2}+m_{3} .
$$

Plurality of harmonics satisfies to this condition, e.g. the 5 th harmonic takes part in a number of resonant interactions: $5=3+2,5=1+4,6=5+1$ and so on.

As a rule, the parametric interaction intensity of waves decreases with increase of their frequencies. But growing SCW is amplified also through the mechanism of two-stream instability. Due to the two-stream instability there is a frequency range (below the optimal frequency $\left.\omega_{\text {opt }}\right)$, in which the growth increment of two-stream instability increases with increase of frequency [22]. Thus, if the frequency of the first SCW harmonic is smaller than the optimal frequency, the higher SCW harmonics will be generated in the two-stream electron beam due to plural three-wave resonant interactions. These harmonics are amplified due to the two-stream instability. As a result, multiharmonic SCW is formed. Its harmonics can have an abnormal spectrum, i.e. higher harmonics can have higher amplitudes.

The second group of plural resonant interactions is related with parametric resonances between harmonics of the electromagnetic signal wave. The relation between the wave number and the frequency can be also considered quasilinear for this wave, when its frequency exceeds beam plasma frequency.

The relation between the wave number and the frequency for this wave can be also considered as quasilinear, when its frequency exceeds plasma frequency of the two-stream beam,

$$
\omega_{1, m}=m \omega_{1,1}, \quad k_{1, m} \approx \omega_{1, m} / c=m \omega_{1,1} / c .
$$

It means that the condition of three-wave parametric interaction between $m_{1}$-th, $m_{2}$-th, and $m_{3}$-th harmonics of the electromagnetic signal, is defined by Eq. (5), as well as for SCW. Thus, for electromagnetic signal wave harmonics the plural three-wave parametric resonant interactions take place, too. Unlike SCW, for the electromagnetic signal wave there is no an additional amplifi- cation mechanism (growing SCW is amplified also due to two-stream instability). Therefore, higher harmonics amplitudes that are generated due to plural three-wave parametric interactions are decreased rapidly with frequency increases.

A third group of plural resonant interactions is associated with the three-wave parametric interactions between the harmonics of the electromagnetic signal wave, the pump and the SCW. For wave number $k_{2, m}=m k_{2,1}$ of the multiharmonic magnetic pumping field the linear dependence on a harmonic number takes place, too $\left(\omega_{2,1}=0\right)$. Therefore, each three $m$-th harmonics of the signal, the pump, and the growing SCW participate in the three-wave resonance in the investigated system. Its condition has the following form:

$$
\omega_{3, m}=\omega_{1, m}, \quad k_{3, m}=k_{1, m}+k_{2, m} .
$$

Equation (7) is the condition of plural parametric resonant interactions between the harmonics of the signal wave, the pump and the SCW.

Thus, the plural three-wave parametric resonant interactions are implemented both in the terminal section and in the modulator. Due to the three-wave parametric resonances the SCW amplification, which results from two-stream instability, is transferred to the electromagnetic signal wave. It allows generating the multiharmonic electromagnetic signal wave with a broad frequency spectrum, in which harmonics have the comparable amplitudes. Superposition of this harmonics can form the powerful ultrashort cluster of electromagnetic field. This paper is dedicated to finding out the conditions of the forming of the powerful ultrashort electromagnetic cluster in multiharmonic two-stream superheterodyne free-electron lasers of a klystron type.

In this paper we construct the nonlinear theory of plural interactions of the signal waves, the pump, and the $\mathrm{SCW}$ in the terminal section and in the modulator, and we analyze the processes in these sections. It should be noted that the obtained system of equations also allows investigating the processes of plural parametric interactions in transit section. To do this, we assume that the pumping wave and the signal wave are missing. As a result, we obtain the opportunity of the analysis of nonlinear wave processes in multiharmonic TSFELs-klystron in general.

\section{The system of cubic-nonlinear equations for complex wave amplitudes}

To carry out non-linear analysis of the multiharmonic processes in TSFEL of a klystron type we obtain the system of truncated equations for amplitudes of waves, which take part in plural three-wave parametric resonances in the terminal section and the modulator.

As initial equations for analysis we use the relativistic quasihydrodynamic equation [21, 23], the continuity equation and Maxwell's equations. Also we take as satisfied the conditions of collisions neglecting and velocity scatter of electrons. We consider the model, in which all values depend only on longitudinal coordinate $z$ and time $t$. 
We divide the solution of the equations into three formally independent stages: (1) solving of the motion problem of two-stream relativistic electron beam in given electromagnetic fields; (2) solving of the continuity equation considering that we already know the velocities of electron beam; (3) solving of the excitation problem of electromagnetic fields, considering velocities and concentrations as known values.

We solve the motion problem and the continuity equation through the use of the method of averaged characteristics [21, 23]. For solving the problem of electromagnetic field excitation we use the method of slowly varying amplitudes. We consider the features of plural threewave parametric resonant interactions of SCWs and the electromagnetic signal during the solving of this problem.

For solving of the motion problem we pass to characteristics of the relativistic quasihydrodynamic equation [21, 23]. The characteristics of these equations are ordinary differential equations. Since we solve the boundary problem, in these equations we pass from the time derivative to coordinate derivative using the well-known relation for the velocity $\mathrm{d} t=\mathrm{d} z / v_{z}$. We include the equations for fast phases $p_{\chi, m}$ in this equation system. We consider that amplitudes of fields change slowly with the change of longitudinal coordinate $z$. We use slow longitudinal coordinate $\zeta=z / \xi$ to describe slow amplitude changes. As a result we get the equation system in standard form

$$
\begin{aligned}
& \frac{\mathrm{d} v_{q x}}{\mathrm{~d} z}=\frac{e\left(E_{x}-\frac{1}{c} v_{q z} B_{y}-\frac{v_{q x}}{c^{2}}\left(v_{q x} E_{x}+v_{q z} E_{z}\right)\right)}{m_{\mathrm{e}} \gamma_{q} v_{q z}}, \\
& \frac{\mathrm{d} v_{q z}}{\mathrm{~d} z}=\frac{e\left(E_{z}+\frac{1}{c} v_{q x} B_{y}-\frac{v_{q z}}{c^{2}}\left(v_{q x} E_{x}+v_{q z} E_{z}\right)\right)}{m_{\mathrm{e}} \gamma_{q} v_{q z}}, \\
& \frac{\mathrm{d} \zeta}{\mathrm{d} z}=\frac{1}{\xi} \\
& \frac{\mathrm{d} p_{q, \chi, m}}{\mathrm{~d} z}=\frac{m \omega_{\chi, 1}}{v_{q z}}-k_{\chi, m} \equiv \Omega_{q, \chi, m} .
\end{aligned}
$$

In these equations $v_{q x}, v_{q z}$ are the velocity vector projections of the $q$-th partial electron beam $\boldsymbol{v}_{q}$ on $X$ and $Z$ axes, respectively; $\gamma_{q}=\left(1-\left(v_{q z}^{2}+v_{q x}^{2}\right) / c^{2}\right)^{-1 / 2}$ is the relativistic factor of the $q$-th beam; $c$ is the light speed in vacuum; $e=-|e|, m_{\mathrm{e}}$ are the charge and the mass of electron, respectively; $E_{x}=E_{1}, B_{y}=B_{1}+B_{2}, E_{z}=E_{3}$, amplitudes of fields $E_{\chi, m}=E_{\chi, m}(\zeta), B_{1, m}=B_{1, m}(\zeta)$ are slowly changing functions of longitudinal coordinate $z$, index $\chi$ takes values 1,3 .

Let us compare system (8)-(10) with the standard $[21,23]$ and write down the vector of slow variables $\boldsymbol{x}$, vector functions $\boldsymbol{X}$, vector of fast phases $\boldsymbol{\psi}$, and vector of phase velocities $\boldsymbol{\Omega}$ in explicit form

$$
\begin{aligned}
& \boldsymbol{x}=\left\{x_{v_{z}}, x_{v_{x}}, x_{\zeta}\right\}=\left\{v_{q z}, v_{q x}, \zeta\right\}, \\
& \frac{1}{\xi} \boldsymbol{X}=\frac{1}{\xi}\left\{X_{v_{z}}, X_{v_{x}}, X_{\zeta}\right\},
\end{aligned}
$$

$$
\begin{gathered}
\frac{1}{\xi} X_{v_{z}}=\frac{e\left[E_{z}+\frac{1}{c} v_{q x} B_{y}-\frac{v_{q z}}{c^{2}}\left(v_{q x} E_{x}+v_{q z} E_{z}\right)\right]}{m_{\mathrm{e}} \gamma_{q} v_{q z}}, \\
\frac{1}{\xi} X_{v_{x}}=\frac{e\left[E_{x}-\frac{1}{c} v_{q z} B_{y}-\frac{v_{q x}}{c^{2}}\left(v_{q x} E_{x}+v_{q z} E_{z}\right)\right]}{m_{\mathrm{e}} \gamma_{q} v_{q z}}, \\
\frac{1}{\xi} X_{\zeta}=\frac{1}{\xi}, \\
\psi=\left\{p_{q, 1,1}, \ldots, p_{q, 1, N}, p_{2,1}, \ldots, p_{2, N}, p_{q, \alpha, 1}, \ldots,\right. \\
\left.p_{q, \alpha, N}, p_{q, \beta, 1}, \ldots, p_{q, \beta, N}, p_{q, \gamma, 1}, \ldots, p_{q, \gamma, N}\right\} \Omega= \\
\left\{\Omega_{q, 1,1}, \ldots, \Omega_{q, 1, N}, \Omega_{2,1}, \ldots, \Omega_{2, N}, \Omega_{q, \alpha, 1}, \ldots,\right. \\
\left.\Omega_{q, \alpha, N}, \Omega_{q, \beta, 1}, \ldots, \Omega_{q, \beta, N}, \Omega_{q, \gamma, 1}, \ldots, \Omega_{q, \gamma, N}\right\} .
\end{gathered}
$$

Further, we use the algorithm of method of averaged characteristics [21] for the case of several fast phases. According to this algorithm we proceed to averaged variables $\bar{x}$ :

$$
\begin{aligned}
& v_{q i}=\bar{v}_{q i}+\sum_{n=1}^{\infty} \frac{u_{v_{i}}^{(n)}(\bar{x}, \boldsymbol{\psi})}{\xi^{n}}, \quad i=(z, x), \\
& \zeta=\bar{\zeta}+\sum_{n=1}^{\infty} \frac{(\bar{x}, \boldsymbol{\psi})}{\xi^{n}} u_{\zeta}^{(n)} .
\end{aligned}
$$

Equations for slow variables have the following form:

$$
\begin{aligned}
& \frac{\mathrm{d} \bar{v}_{q z}}{\mathrm{~d} z}=\sum_{n=1}^{\infty} \frac{1}{\xi^{n}} A_{v_{z}}^{(n)}(\bar{x}), \quad \frac{\mathrm{d} \bar{v}_{q x}}{\mathrm{~d} z}=\sum_{n=1}^{\infty} \frac{1}{\xi^{n}} A_{v_{x}}^{(n)}(\bar{x}), \\
& \frac{\mathrm{d} \bar{\zeta}}{\mathrm{d} z}=\sum_{n=1}^{\infty} \frac{1}{\xi^{n}} A_{\zeta}^{(n)}(\bar{x}) .
\end{aligned}
$$

We restrict ourselves by the third approximation for $1 / \xi$. The algorithm of finding out $\boldsymbol{u}^{(n)}$ and $\boldsymbol{A}^{(n)}$ is known and described, e.g., in [21]. Particularly from these formulae it follows that $u_{\zeta}^{(n)}=0$ for any $n ; A_{\zeta}^{(1)}=1, A_{\zeta}^{(n)}=0$ for

$$
\begin{aligned}
& n>1 ; A_{v_{z}}^{(1)}=A_{v_{z}}^{(2)}=0, A_{v_{x}}^{(1)}=A_{v_{x}}^{(2)}=0, \\
& \frac{1}{\xi} u_{v_{z}}^{(1)}=\frac{e}{m_{\mathrm{e}} \bar{v}_{q z}}\left(1-\frac{\bar{v}_{q z}^{2}}{c^{2}}\right)^{3 / 2} \\
& \quad \times \sum_{\chi}^{\alpha, \beta, \gamma} \sum_{m=1}^{N}\left[\frac{E(\bar{\zeta})_{\chi, m}}{\mathrm{i} \Omega_{q, \chi, m}} \exp \left(\mathrm{i} p_{q, \chi, m}\right)+\text { c.c. }\right], \\
& \frac{1}{\xi} u_{v_{x}}^{(1)}=\frac{e}{m_{\mathrm{e}} \bar{\gamma}_{q}} \sum_{m=1}^{N}\left[\left(\frac{E(\bar{\zeta})_{1, m}}{\mathrm{i} \Omega_{q, 1, m} \bar{v}_{q z}}-\frac{B(\bar{\zeta})_{1, m}}{\mathrm{i} \Omega_{q, 1, m} c}\right)\right. \\
& \left.\quad \times \exp \left(\mathrm{i} p_{1, m}\right)-\frac{B_{2, m}}{\mathrm{i} \Omega_{2, m} c} \exp \left(\mathrm{i} p_{2, m}\right)+\text { c.c. }\right],
\end{aligned}
$$

and so on. As a result, we obtain the solution both for the oscillating velocity component and for non-oscillating velocity component (13)

We obtain the solution of the continuity equation in the same way as in the case of the motion problem. To obtain solutions of the electromagnetic field excitation problem, we substitute the expressions for the velocity (12) and the concentration of partial beams into Maxwell's equations (7). We consider that these expressions obtained 
through the use of method of averaged characteristics have the form of series in the small parameter $1 / \xi$. We also expand it in Fourier series in the fast phase harmonics. As a result of calculation procedures we obtain a system of differential equations in a cubic nonlinear approximation for the amplitudes of the electric fields of the electromagnetic signal wave and the space-charge wave

$$
\begin{gathered}
K_{2,1, m} \frac{\mathrm{d}^{2} E_{1, m}}{\mathrm{~d} z^{2}}+K_{1,1, m} \frac{\mathrm{d} E_{1, m}}{\mathrm{~d} z}+D_{1, m} E_{1, m}= \\
K_{3,1, m} E_{\alpha, m} B_{2, m}+F_{1, m}, \\
C_{2,3, m} \frac{\mathrm{d}^{2} E_{3, m}}{\mathrm{~d} z^{2}}+C_{1,3, m} \frac{\mathrm{d} E_{3, m}}{\mathrm{~d} z}+D_{3, m} E_{3, m}= \\
C_{3,3, m} E_{1, m} B_{2, m}^{*}+C_{4, m}\left\langle\int E_{3}\left(\int E_{3} \mathrm{~d} p_{3,1}\right) \mathrm{d} p_{3,1}\right\rangle_{p_{3, m}} \\
+C_{5, m}\left\langle\left(\int E_{3} \mathrm{~d} p_{3,1}\right)^{2}\right\rangle_{p_{3, m}}+F_{3, m} .
\end{gathered}
$$

In these equations

$$
\begin{aligned}
& D_{1, m}=k_{1, m}^{2}-\frac{\omega_{1, m}^{2}}{c^{2}}-\sum_{q=1}^{2} \frac{\omega_{\mathrm{p}}^{2}}{\bar{\gamma}_{q} c^{2}}, \\
& D_{3, m}=-\mathrm{i} k_{3, m}\left(1-\sum_{q=1}^{2} \frac{\omega_{\mathrm{p}, q}^{2}}{\Omega_{3, q, m}^{2} \bar{v}_{q z}^{2} \bar{\gamma}_{q}^{3}}\right)
\end{aligned}
$$

are the dispersion functions of the electromagnetic signal wave and the SCW, respectively, for $m$-th harmonic; $F_{3, m}=4 \pi e\left\langle\sum_{q=1}^{2} \frac{1}{\xi^{3}} u_{n, q}^{(3)}\right\rangle_{p_{3, m}}, \quad F_{1, m}=$ $\frac{4 \pi e}{c}\left\langle\sum_{q=1}^{2} \frac{1}{\xi^{3}}\left(\bar{n}_{q} u_{v_{x}, q}^{(3)}+u_{n, q}^{(1)} u_{v_{x}, q}^{(2)}+u_{n, q}^{(2)} u_{v_{x}, q}^{(1)}\right)\right\rangle_{p_{1, m}} \quad$ are functions containing the cubic non-linear components, that are also related to parametric resonant interactions in the investigated system. Functions $u_{n, q}^{(1)} / \xi, u_{n, q}^{(2)} / \xi^{2}$, $u_{n, q}^{(3)} / \xi^{3}$ define the oscillatory components of the partial beam concentration in the first, the second and the third approximation respectively; $u_{v_{x}, q}^{(1)} / \xi, u_{v_{x}, q}^{(2)} / \xi^{2}, u_{v_{x}, q}^{(3)} / \xi^{3}$ are the oscillatory components of velocity $v_{x}(12), \delta_{m, 1}$ is the Kronecker delta

$$
\begin{aligned}
& K_{1,1, m}=\frac{\partial D_{1, m}}{\partial\left(-\mathrm{i} k_{1, m}\right)}, \quad K_{2,1, m}=\frac{1}{2} \frac{\partial^{2} D_{1, m}}{\partial\left(-\mathrm{i} k_{1, m}\right)^{2}}, \\
& K_{3,1, m}=-\sum_{q=1}^{2} \frac{\omega_{\mathrm{p}, q}^{2} \omega_{1, m} e}{2 \Omega_{3, q, m} m_{\mathrm{e}} \bar{\gamma}_{q}^{4} k_{1, m} k_{2, m} c^{3} \bar{v}_{q z}^{2}} \\
& \quad \times\left(\frac{\bar{v}_{q z}^{2} \bar{\gamma}_{q}^{2}}{c^{2}}-\frac{k_{3, m}}{\Omega_{3, q, m}}\right), \\
& D_{3, m}=-\mathrm{i} k_{3, m}\left(1-\sum_{q=1}^{2} \frac{\omega_{\mathrm{p}, q}^{2}}{\Omega_{3, q, m}^{2} \bar{v}_{q z}^{2} \bar{\gamma}_{q}^{3}}\right), \\
& C_{1,3, m}=\frac{\partial D_{3, m}}{\partial\left(-\mathrm{i} k_{3, m}\right)},
\end{aligned}
$$

$$
\begin{aligned}
& C_{2,3, m}=\frac{1}{2} \frac{\partial^{2} D_{3, m}}{\partial\left(-\mathrm{i} k_{3, m}\right)^{2}}, \\
& C_{3,3, m}=-\sum_{q=1}^{2}\left[\frac{\omega_{\mathrm{p}, q}^{2} e k_{3, m}}{\bar{v}_{q z}^{2} \Omega_{3, q, m}^{2} m_{\mathrm{e}} \bar{\gamma}_{q}^{2} c^{2} k_{2, m}}\right. \\
& \left.\times\left(\frac{\bar{v}_{q z}}{c}-\frac{k_{3, m} c}{\omega_{3, m}}\right)\right], \\
& C_{4, m}=\sum_{q=1}^{2} \frac{\omega_{\mathrm{p}, q}^{2} k_{3,1}}{c \bar{\gamma}_{q}^{4} \bar{v}_{q z}^{4} \Omega_{3, q, 1}^{3}} \frac{e}{m_{\mathrm{e}}} \\
& \times\left(\frac{2 k_{3,1}+\omega_{3,1} / \bar{v}_{q z}}{\Omega_{3, q, 1} \bar{\gamma}_{q}^{2}}-3 \frac{\bar{v}_{q z} \omega_{3,1} / k_{3,1}}{c^{2}}\right), \\
& C_{5, m}=\sum_{q=1}^{2}\left(\frac{\omega_{\mathrm{p}, q}^{2}}{c \bar{\gamma}_{q}^{6}} \frac{e}{m_{\mathrm{e}}} \frac{k_{3,1}}{\bar{v}_{q z}^{4} \Omega_{3, q, 1}^{3}}\right), \\
& \omega_{\mathrm{p}, q}^{2}=4 \pi \bar{n}_{q} e^{2} / m_{\mathrm{e}} .
\end{aligned}
$$

Coefficients in the system (14) also depend on the slowly varying components of the velocities $\bar{v}_{q}$ and the partial beam concentrations $\bar{n}_{q}$. Therefore we supplement the system (14) with equations for these slowly varying components

$$
\begin{aligned}
& \frac{\mathrm{d} \bar{v}_{q z}}{\mathrm{~d} z}=V_{q}\left(\boldsymbol{E}_{1}, \boldsymbol{B}_{1}, \boldsymbol{B}_{2}, \boldsymbol{E}_{3}, \bar{v}_{q}, \bar{n}_{q}\right), \\
& \frac{\mathrm{d} \bar{n}_{q}}{\mathrm{~d} z}=N_{q}\left(\boldsymbol{E}_{1}, \boldsymbol{B}_{1}, \boldsymbol{B}_{2}, \boldsymbol{E}_{3}, \bar{v}_{q}, \bar{n}_{q}\right),
\end{aligned}
$$

where functions $V_{q}=\sum_{q=1}^{2} \frac{1}{\xi^{3}} A_{v_{z}, q}^{(3)}, N_{q}=\sum_{q=1}^{2} \frac{1}{\xi^{3}} A_{n, q}^{(3)}$ contain cubic non-linear components.

We analyze the obtained system (14)-(18) numerically. This equation system describes non-linear processes in the TSFEL of a klystron type.

\section{Higher harmonics influence on the wave dynamics in TSFEL in maximum amplification mode}

Let us analyze the influence of the plural three-wave interactions on the dynamics of first harmonic of TSFEL in the mode of maximum signal amplification. For this purpose we consider the one-section TSFEL with H-ubitron pumping, which is described by the theoretical model represented in Fig. 2 and by the equation system (14)-(18). Figures 3 and 4 illustrate the wave dynamics in such system.

Parameters of investigated TSFEL are chosen as following: the plasma frequency of electron partial beams $\omega_{\mathrm{p}}=1.5 \times 10^{11} \mathrm{~s}^{-1}$; the average value of relativistic factor $\gamma_{0}=4.5$; the difference of partial beam relativistic factors $\Delta \gamma=0.5$; the induction of pumping magnetic field $B_{2,1}=400$ Gs. We assume that pump magnetic field is monochromatic. The wavelength of the first signal harmonic is $\lambda_{1,1}=0.04 \mathrm{~cm}$ and the undulation period of the pump magnetic field is $\lambda_{2,1}=1.56 \mathrm{~cm}$. In the case of maximum amplification the first harmonic frequency 


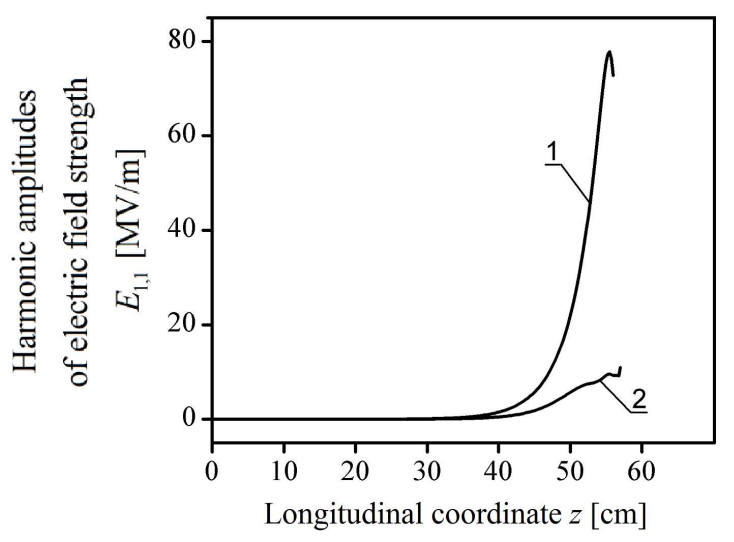

Fig. 3. Amplitudes of the first harmonic of the signal wave electric field as a function of the longitudinal coordinate $z$.

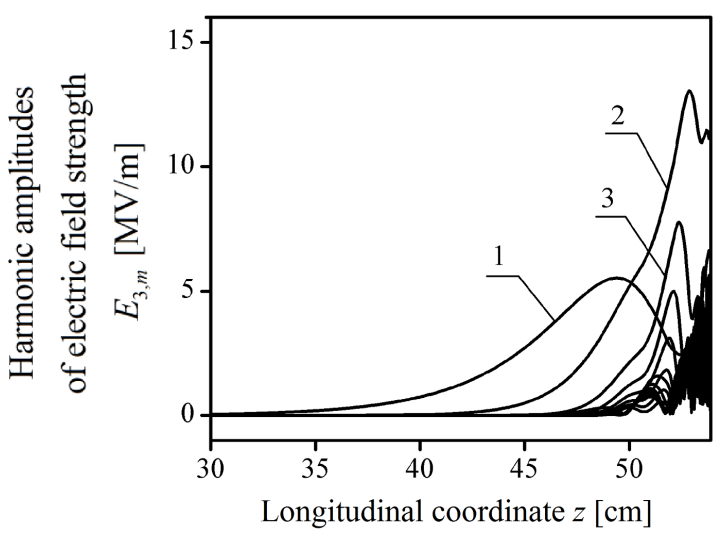

Fig. 4. Amplitudes of the SCW electric field harmonics as a function of the longitudinal coordinate $z$.

of the electromagnetic signal, which is supplied to the input of the TSFEL, is equal to the optimal frequency $\omega_{1,1}=\omega_{\text {opt }}=\sqrt{3} \omega_{\mathrm{p}} \gamma_{0}^{3 / 2}\left(1-\gamma_{0}^{-2}\right) / \Delta \gamma$.

Figure 3 shows the amplitudes of the first harmonic of the signal wave electric field as a function of the longitudinal coordinate $z$ of TSFEL. Curve 1 in this figure describes the case when we take into account only the first harmonics of interacting waves. Curve 2 refers to the case when we consider 50 harmonics. As we can see, in the case of considering of three-wave plural resonances the saturation level of the electromagnetic signal wave decreases eight times when compared to the case of neglecting of higher harmonics. It indicates that consideration of higher harmonics in two-stream SFEL is necessary.

The significant influence of higher harmonics on the signal wave saturation level is explained by the generation of SCW higher harmonics and by the decrease of the first harmonic amplitude of SCW due to this process. The first SCW harmonic, in turn, is related with the first harmonic electromagnetic signal by three-wave parametric resonance. Figure 4 shows the dynamics of ten harmonics of the SCW. As we can see, the saturation levels of 2nd (position 2) and 3rd (position 3) harmonics exceed the saturation level of 1st harmonic (position 1).
The saturation levels of other harmonics are comparable with the saturation level of the first harmonic.

\section{Features of ultrashort electromagnetic field clusters forming}

Let us consider the features of the forming of ultrashort electromagnetic field clusters in TSFEL. It is clear that in the ideal case we should obtain a periodic set of deltafunctions at the output of the multiharmonic TSFEL. These functions can have, e.g., the following form:

$$
E(t)=A T[-\delta(t-T / 4+n T)+\delta(t-3 T / 4+n T)] .
$$

In this formula $\delta$ is a Dirac delta-function, $T$ is a period, $n$ is an integer, $A$ is a multiplier. At its core, such sequence of delta-functions is a complex periodic multiharmonic signal, and it can be expanded in a Fourier series

$$
E(t)=E_{0}+\sum_{m=1}^{\infty}\left(E_{m} \exp (\mathrm{i} m(2 \pi / T) t)+\text { c.c. }\right),
$$

where

$$
E_{m}=(1 / T) \int_{-T / 2}^{T / 2} E(t) \exp (-\mathrm{i} m(2 \pi / T) \tau) \mathrm{d} \tau
$$

is the complex amplitude of $m$-th harmonic. For the investigated case, when $E(t)$ is defined by relation (19), the complex amplitudes $E_{m}$ can be easily obtained through the use of expression (21). As a result we get

$$
E_{m}=2 \mathrm{i} A \sin (m \pi / 2),
$$

i.e., $E_{0}=0, E_{1}=+2 \mathrm{i} A, E_{2}=0, E_{3}=-2 \mathrm{i} A, E_{4}=0$, $E_{5}=+2 \mathrm{i} A, E_{6}=0, E_{7}=-2 \mathrm{i} A$ and so on. Equation (22) implies as follows: the amplitudes of all even harmonics are zero; the absolute values of odd harmonics are the same and equal to $2 A$; phases of the 1 st, 5 th, 9 th, etc. harmonics are equal to $(+\pi / 2)$; the phases of the $3 \mathrm{rd}, 7 \mathrm{th}, 11 \mathrm{th}$, etc. harmonics are equal to $(-\pi / 2)$. Therefore, to obtain the series of femtosecond clusters of the electromagnetic field (19) we should form the multiharmonic signal with amplitudes and phases satisfying the condition (22).

We investigate the dynamics of the forming of powerful ultrashort electromagnetic clusters in the TSFEL, using Eqs. (14)-(18) with parameters given in Table I. We investigate the case, when the monochromatic signal is supplied to the input of the TSFEL-former.

For the investigated system the critical frequency is $\omega_{\mathrm{cr}}=8.63 \times 10^{13} \mathrm{~s}^{-1}$, the optimal frequency is $\omega_{\mathrm{opt}}=$ $5.3 \times 10^{13} \mathrm{~s}^{-1}$. The frequency of the first harmonic of the electromagnetic signal is $\omega_{1,1}=2.7 \times 10^{12} \mathrm{~s}^{-1}$, which is 32 times smaller than the critical frequency. Comparing the first harmonic frequency with the plasma frequency of a two-stream beam $\sqrt{2} \omega_{\mathrm{p}}=1.8 \times 10^{11} \mathrm{~s}^{-1}$, we see that the condition $\omega_{1,1} \gg \sqrt{2} \omega_{\mathrm{p}}$ is satisfied. It means that the frequency of the first harmonic of the electromagnetic signal significantly exceeds the two-stream electron beam plasma frequency. Therefore, the dependence of the wave number on the frequency can be considered as linear for 
TABLE I

Parameters TSFEL of the klystron type.

\begin{tabular}{c|c}
\hline \hline Parameter & Value \\
\hline wavelength of the first harmonic & 0.7 \\
of the signal wave $\left(\lambda_{1,1}\right)[\mathrm{mm}]$ & 7.5 \\
average value of the relativistic factor $\left(\gamma_{0}\right)$ & 0.07 \\
relativistic factors difference $(\Delta \gamma)$ & $1.3 \times 10^{11}$ \\
plasma frequency of partial beams & 6.5 \\
$\left(\omega_{p}\right)\left[\mathrm{s}^{-1}\right]$ & 1.0 \\
undulator period $\left(\lambda_{2,1}\right)[\mathrm{cm}]$ & 0.5 \\
modulator section length $[\mathrm{m}]$ & 1.5 \\
transit section length $[\mathrm{m}]$ &
\end{tabular}

all harmonics of the signal wave. Thus, there are 32 SCW harmonics in the frequency domain, in which the growth takes place due to the two-stream instability. This frequency domain is limited by the critical frequency $\omega_{\mathrm{cr}}$. Therefore, in further calculations we take into account the 50 harmonics of each type of the waves involved in the parametric resonance interactions.

\section{Forming of electromagnetic field clusters in TSFEL}

We consider the model presented in Fig. 1. The monochromatic signal is supplied to the input of modulation section 3 of TSFEL-klystron. We use the system of Eqs. (14)-(18) for the analysis of cluster forming.

In the modulator (position 3, Fig. 1, see also Fig. 2) the SCW is excited in the two-stream electron beam as a result of the three-wave parametric resonance between electromagnetic signal 1 and the H-ubitron field of modulator 3. Since the monochromatic signal wave is supplied to the modulator input, the H-ubitron magnetic field of the modulator is also taken as monochromatic. We supply the signal with amplitude $100 \mathrm{~V} / \mathrm{cm}$ on the modulator input, other harmonics are zero. We take the induction of magnetic H-ubitron field as $B_{2,1}=1000$ Gs, the modulator period is $\lambda_{2,1}=6.5 \mathrm{~cm}$. On leaving from modulator 3 the SCW has the spectrum which is shown in Fig. 5. As we can see from Fig. 5, in modulator 3 it is formed the SCW in which amplitude of the first harmonic is maximal and the amplitudes of higher harmonics are smaller than the amplitude of the first harmonic.

Further, the SCW excited in two-stream electron beam grows due to the two-stream instability. Since the frequency of the first harmonic of SCW is 32 times smaller than critical frequency then all the first 32 harmonics are amplified due to the two-stream instability. For the harmonics whose frequency is smaller than the optimum frequency (20th harmonic corresponds to the optimal frequency), their growth rates increase with enhancing their frequency. As a result, the abnormal spectrum shown in Fig. 6 begins to form at the output of the transit section $(z=150 \mathrm{~cm})$. In this case the maximal amplitude of the spectrum corresponds to the second harmonic (Fig. 6), but not the first one as in the case of Fig. 5.

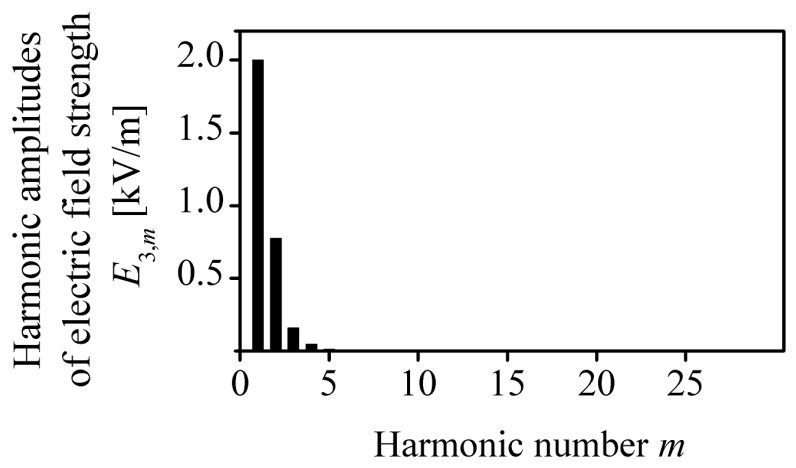

Fig. 5. SCW spectrum at the output from modulator 3 $(z=100 \mathrm{~cm})$.

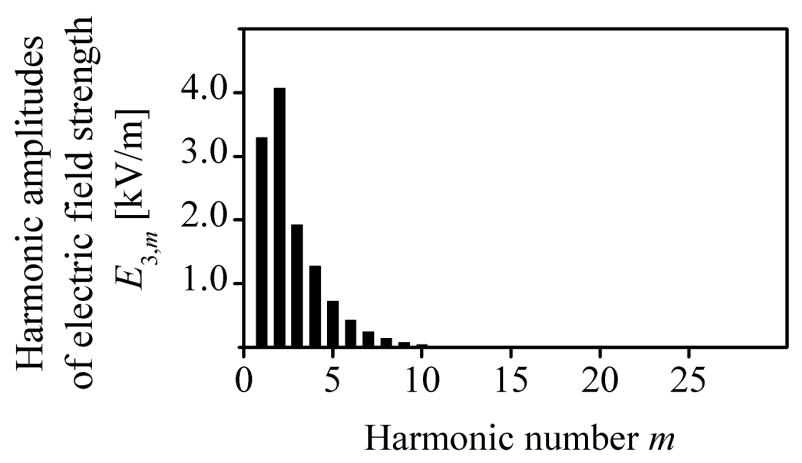

Fig. 6. SCW spectrum at the output from the transit section $(z=150 \mathrm{~cm})$.

The dependence of the initial phases of SCW harmonics in the modulator and the transit section on the longitudinal coordinate $z$ is shown in Fig. 7. As we can see, the transient processes take place at the initial stage of the excitation of the multiharmonic SCW. At the output of the transit section the harmonic phases already have stationary values. It should be noted that the phases difference between adjacent harmonics is practically the same and equal to $\approx 0.6 \pi$.

It should also be noted that abnormal spectrum is not formed yet at the output of the transit section. Its forming continues in the terminal section, too. The excitation of the electromagnetic wave occurs in this section simultaneously. It happens due to plural three-wave parametric resonances between the multiharmonic SCW and

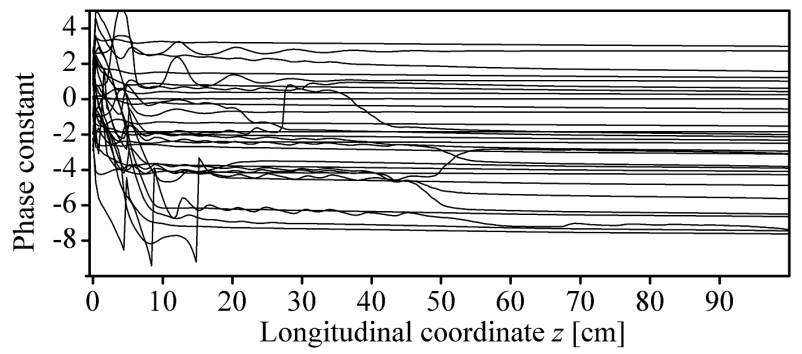

Fig. 7. Initial phases of SCW harmonics in the modulator and the transit section as a function of the longitudinal coordinate $z$. 


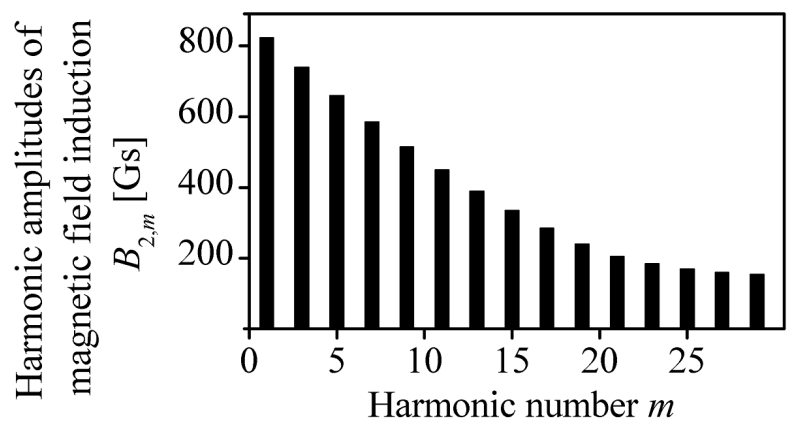

Fig. 8. Harmonic amplitudes $B_{2, m}$ of magnetic field induction in the terminal section as a function of the harmonic number $m$.

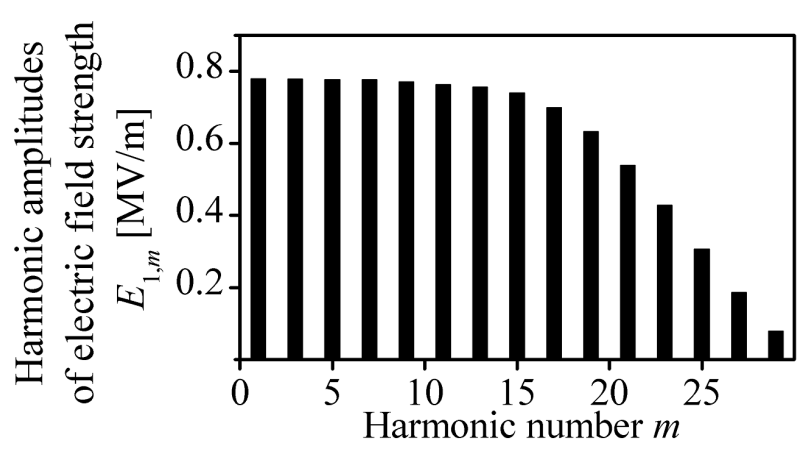

Fig. 9. Harmonic amplitudes $E_{1, m}$ of electromagnetic signal wave strength as a function of the harmonic number $m$ for longitudinal coordinate $z=257 \mathrm{~cm}$.

the multiharmonic H-ubitron pumping field. We choose the amplitude and the initial phase of the multiharmonic magnetic pumping field harmonics in such a way as to form the ultrashort multiharmonic electromagnetic cluster in the terminal section. Amplitudes and phases of such wave have to satisfy the conditions: the amplitudes of all even harmonics are zero; the absolute values of odd harmonics have almost similar values; the phases of the 1 st, 5th, 9th, etc. harmonics are equal to $(+\pi / 2)$; the phases of the $3 \mathrm{rd}, 7 \mathrm{th}, 11 \mathrm{th}$, etc. harmonics are equal to $(-\pi / 2)$. The spectrum of such magnetic field is shown in Fig. 8, spectrum of the electromagnetic cluster in point $z=257 \mathrm{~cm}$ of the system is shown in Fig. 9 .

Figure 10 shows the dependence of the amplitudes of the signal wave $E_{1, m}$ on longitudinal coordinate $z$. We can see that amplitudes of odd harmonics grow exponentially, and have practically the same values in the region of the coordinate $z=257 \mathrm{~cm}$.

Figure 11 shows the dependences of the initial phases of the electromagnetic wave harmonics on the longitudinal coordinate $z$. As we can see, near the longitudinal coordinate $z=257 \mathrm{~cm}$ the initial phases of the 1st, 5th, 9 th, etc. harmonics are approximately equal to $(+\pi / 2)$ (curves 1 in Fig. 11), and the initial phases of the 3rd, 7 th, 11th, etc. harmonics are equal to $(-\pi / 2)$, respectively (curves 2 in Fig. 11).

Thus, two-stream FEL forms the electromagnetic field cluster in point $z=257 \mathrm{~cm}$. The harmonics of this

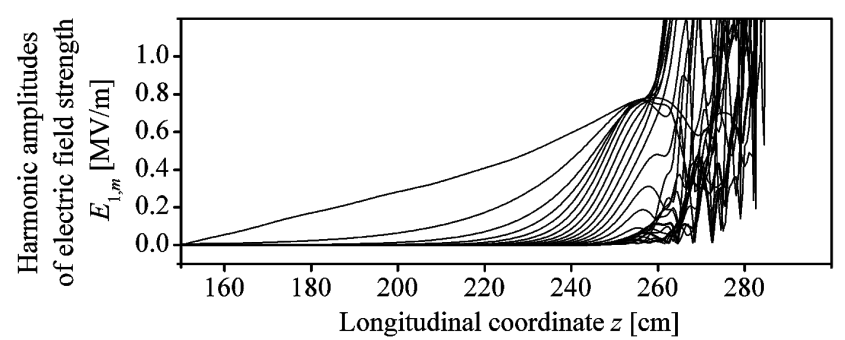

Fig. 10. Harmonic amplitudes $E_{1, m}$ of electromagnetic signal wave strength as a function of longitudinal coordinate $z$.

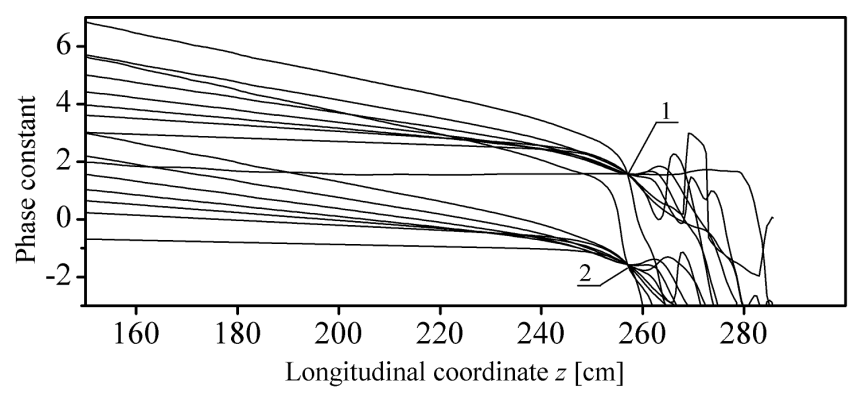

Fig. 11. Initial phases of signal wave harmonics as a function of longitudinal coordinate $z$ of the terminal section. Curves 1 correspond to the 1st, 5th, 9 th, etc. harmonics, curves 2 correspond to the $3 \mathrm{rd}$, 7th, 11th, etc. harmonics.

cluster satisfy the condition (22). Figure 12 shows the dependence of electromagnetic signal energy flux density on time normalized at the first harmonic period. As expected, the electromagnetic signal has the form of the short cluster with duration $\tau_{\mathrm{p} 2}=4 \times 10^{-14} \mathrm{~s} \approx$ $40 \mathrm{fs}$. If we accept the electric current of partial electron beam as equal to $1.1 \mathrm{kA}$, then the instantaneous power of such cluster is $1 \mathrm{MW}$. The average power of such electromagnetic cluster is $16 \mathrm{~kW}$. Therefore, multiharmonic TSFEL can form the powerful ultrashort clusters of an electromagnetic field.

As we have mentioned above, to form the series of femtosecond clusters of the electromagnetic field we have to form multiharmonic signal with amplitudes and phases satisfying the conditions: the amplitudes of all even harmonics are zero; the absolute values of odd harmonics are the same; phases of the 1st, 5th, 9th, etc. harmo-

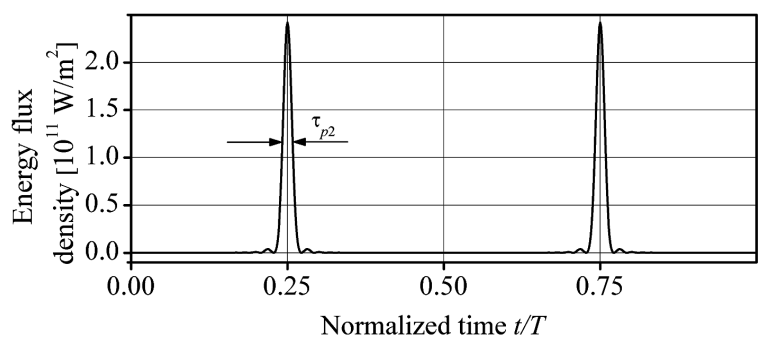

Fig. 12. Electromagnetic signal energy flux density as a function of normalized time $t / T$ in the terminal section with $z=257 \mathrm{~cm}$. 
nics are equal to $(+\pi / 2)$; the phases of the $3 \mathrm{rd}, 7 \mathrm{th}$, 11 th, etc. harmonics are equal to $(-\pi / 2)$. As follows from Fig. 10 and Fig. 11, these conditions are satisfied in point $z=257 \mathrm{~cm}$. In other points these conditions are violated and the electromagnetic cluster form is changed in comparison with the cluster presented in Fig. 12. Thus, on $z=250 \mathrm{~cm}$ the maximal value of energy flux density is decreased twice and the width of the electromagnetic cluster is increased by $\approx 15 \%$. In this point long-wave low frequency components appear. At $z=260 \mathrm{~cm}$ the maximal value of energy flux density is almost the same as in Fig. 12, but the cluster width is increased 1.8 times. In this point high frequency oscillations appear. Therefore, in the coordinate range from $z=250 \mathrm{~cm}$ to $z=260 \mathrm{~cm}$ the cluster with maximal energy flux density value of electromagnetic signal from $2.4 \times 10^{11} \mathrm{~V} / \mathrm{m}^{2}$ to $1.2 \times 10^{11} \mathrm{~V} / \mathrm{m}^{2}$ and cluster width from 40 fs to $75 \mathrm{fs}$ is formed.

It should also be noted that the process of electromagnetic cluster formation is sufficiently sensitive to the parametric variations of the terminal section first of all. The analysis of the cluster form dependence on the system parameters will be carried out in other works.

\section{Conclusions}

Therefore, in this paper we have developed the cubic non-linear self-consistent theory of multiharmonic twostream free electron lasers of a klystron type designed for forming of the powerful ultrashort electromagnetic clusters. We have taken into account the plural three-wave parametric resonant interactions. We have studied the influence of the plural three-wave interactions of the growing SCW harmonics on the dynamics of main harmonic of TSFEL working in the mode of maximal powerful signal amplification. We have shown that accounting of such interactions can lead to the decrease of the saturation level of an electromagnetic signal eight times and more. It indicates that accounting of SCW higher harmonics is necessary for the analysis of the TSFEL operation. The operation mode in which the first TSFEL harmonic is much less the critical frequency of the twostream instability is used for the forming of the powerful ultrashort electromagnetic clusters. We have carried out the amplitude, phase and spectral analyses of the processes of the forming of the ultrashort electromagnetic clusters in the TSFEL. The conditions that are necessary for the forming of short electromagnetic clusters are specified. The fundamental opportunity to create the ultrashort electromagnetic clusters in the multiharmonic TSFEL is demonstrated. It is shown that the forming of the clusters with duration $\approx 40 \mathrm{fs}$ is possible in the TSFEL with beam energy $\approx 3.3 \mathrm{MeV}$. These devices can have wide practical applications as sources of powerful broadband electromagnetic signals.

\section{References}

[1] G. Bekefi, K.D. Jacobs, J. Appl. Phys. 53, 4113 (1982).

[2] M. Botton, A. Ron, IEEE Trans. Plasma Sci. 18, 416 (1990).

[3] M. Botton, A. Ron, J. Appl. Phys. 67, 6583 (1990).

[4] H. Wilhelmsson, Phys. Scr. 44, 603 (1991).

[5] T.A. Davidova, H. Wilhelmsson, Phys. Scr. 45, 607 (1992).

[6] G. Bekefi, J. Appl. Phys. 71, 4128 (1992).

[7] C. Chen, P. Catravas, G. Bekefi, Appl. Phys. Lett. 62, 1579 (1993).

[8] V.V. Kulish, S.A. Kuleshov, A.V. Lysenko, Int. J. Infrared Millim. Waves 14, 451 (1993).

[9] V.V. Kulish, S.A. Kuleshov, A.V. Lysenko, Int. J. Infrared Millim. Waves 15, 77 (1994).

[10] V.V. Kulish, A.V. Lysenko, V.I. Savchenko, Int. J. Infrared Millim. Waves 24, 285 (2003).

[11] V.V. Kulish, O.V. Lysenko, V.I. Savchenko, I.G. Majornikov, Laser Phys. 15, 1629 (2005).

[12] B.W.J. McNeil, G.R.M. Robb, M.W. Poole, Phys. Rev. E 70, 035501 (2004).

[13] W. Liu, Z. Yang, Z. Liang, Int. J. Infrared Millim. Waves 27, 1073 (2006).

[14] H. Mehdian, N. Abbasi, Phys. Plasmas 15, 013111 (2008).

[15] N. Mahdizadeh, F.M. Aghamir, J. Appl. Phys. 113 083305 (2013).

[16] S. Saviz, M. Karimi, Chin. Phys. B 23, 034103 (2014).

[17] T. Mohsenpour, N. Mehrabi, Phys. Plasmas 20, 082133 (2013).

[18] S. Nadrifard, B. Maraghechi, T. Mohsenpour, Plasma Phys. Control. Fusion 55, 025012 (2013).

[19] King-Yuen Ng, Physics of Intensity Dependent Beam Instabilities, World Sci., Singapore 2006.

[20] N.A. Krall, A.W. Trivelpiece, Principles of Plasma Physics, San Francisco Press, New York 1986.

[21] V.V. Kulish, Hierarchic Electrodynamics and Free Electron Lasers, CRC Press, Taylor \& Francis Group, 2011.

[22] V.V. Kulish, A.V. Lysenko, M.Yu. Rombovsky, Plasma Phys. Rep. 36, 594 (2010).

[23] V.V. Kulish, A.V. Lysenko, A.Ju. Brusnik, J. Infrared Millim. TeraHz Waves 33, 149 (2012).

[24] J.C. Diels, W. Rudolph, Ultrashort Laser Pulse Phenomena, Academic Press, New York 2006.

[25] M. Braun, P. Gilch, W. Zinth, Ultrashort Laser Pulses in Biology and Medicine, Springer, Berlin 2008. 